\title{
The reliability and validity study of the Kinesthetic and Visual Imagery Questionnaire in individuals with Multiple Sclerosis
}

\author{
Yousef Moghadas Tabrizi ${ }^{1,2}$, Nasser Zangiabadi ${ }^{1}$, Shahrzad Mazhari ${ }^{1}$, \\ Farzaneh Zolala $^{3}$
}

\begin{abstract}
Objective: Motor imagery (MI) has been recently considered as an adjunct to physical rehabilitation in patients with multiple sclerosis (MS). It is necessary to assess MI abilities and benefits in patients with MS by using a reliable tool. The Kinesthetic and Visual Imagery Questionnaire (KVIQ) was recently developed to assess MI ability in patients with stroke and other disabilities. Considering the different underlying pathologies, the present study aimed to examine the validity and reliability of the KVIQ in MS patients. Method: Fifteen MS patients were assessed using the KVIQ in 2 sessions (5-14days apart) by the same examiner. In the second session, the participants also completed a revised MI questionnaire (MIQ-R) as the gold standard. Intra-class correlation coefficients (ICCs) were measured to determine test-retest reliability. Spearman's correlation analysis was performed to assess concurrent validity with the MIQ-R. Furthermore, the internal consistency (Cronbach's alpha) and factorial structure of the KVIQ were studied. Results: The test-retest reliability for the KVIQ was good (ICCs: total KVIQ=0.89, visual KVIQ=0.85, and kinesthetic $\mathrm{KVIQ}=0.93$ ), and the concurrent validity between the KVIQ and MIQ-R was good (r=0.79). The KVIQ had good internal consistency, with high Cronbach's alpha (alpha=0.84). Factorial analysis showed the bi-factorial structure of the KVIQ, which was explained by visual $=57.6 \%$ and kinesthetic $=32.4 \%$. Conclusions: The results of the present study revealed that the KVIQ is a valid and reliable tool for assessing MI in MS patients.
\end{abstract}

Keywords: multiple sclerosis; motor imagery; KVIQ.

\section{HOW TO CITE THIS ARTICLE}

Moghadas Tabrizi Y, Zangiabadi N, Mazhari S, Zolala F. The reliability and validity study of the Kinesthetic and Visual Imagery Questionnaire in individuals with Multiple Sclerosis. Braz J Phys Ther. 2013 Nov-Dec; 17(6):588-592. http://dx.doi.org/10.1590/ S1413-35552012005000124

\section{- Introduction}

Mental imagery is defined as the mental invention or recreation of an experience that resembles the actual perception of an object or an event ${ }^{1}$. Motor imagery (MI) is a specific type of mental imagery that involves the mental rehearsal of a motor act without overt movement output ${ }^{2}$. MI may be performed using different modalities (kinesthetic and visual) from either a first- or third-person perspective. In the present study, we focused on the first-person perspective, that is, self-visualizing oneself in an action (visual) or implying some esthetic sensation elicited by an action ${ }^{3}$. MI is a powerful tool and has been used in non-disabled individuals to facilitate motor skill learning 4 . Several studies have shown the effectiveness of MI as a potential adjunct to physical rehabilitation in some neurological diseases (post-stroke ${ }^{5}$, Parkinson's disease ${ }^{6}$, and more recently multiple sclerosis ${ }^{7}$.
Mental practice is the systematic and repetitive use of imagery. A major difficulty when using mental practice through MI is to determine the extent to which a person can generate mental representations of movements. Some patients may not be able to engage in MI, hence they may not benefit from mental practice ${ }^{8}$. Therefore, it is important to assess MI abilities in various diseases. Impaired MI abilities have been determined using behavioral tasks in patients with neurological diseases that affect the parietal cortex and prefrontal area9. These findings reveal the limitation of MI as a therapeutic tool. In fact, if patients are unable to engage in MI correctly, this method is unlikely to be effective.

Multiple sclerosis (MS) is a demyelinating disease of the central nervous system that causes functional neuronal disconnections, leading to motor as well as cognitive dysfunctions ${ }^{10}$. There are some theoretical

\footnotetext{
${ }^{1}$ Neuroscience Research Center, Kerman University of Medical Sciences, Kerman, Iran

${ }^{2}$ Faculty of Physical Education, Tehran University, Tehran, Iran

${ }^{3}$ Faculty of Health, Kerman University of Medical Sciences, Kerman, Iran

Received: 01/18/2013 Revised: 06/24/2013 Accepted: 07/05/2013
} 
reasons to suppose that MI may be affected in MS patients. Functional magnetic resonance imaging (fMRI) studies of the motor system of MS patients have shown abnormal responses in several cortical areas, including the sensorimotor cortex, parietal lobe, and supplementary motor area (common areas involved in MI) ${ }^{11}$. Studies have recently revealed impairment of MI ability in MS patients ${ }^{7}$. Although the vividness of imagery is preserved in these patients, the accuracy and temporal organization of MI are affected. These results suggest that some patients with MS may not be able to engage in motor imagery, hence they may not benefit from mental practice. Therefore, it is important to assess MI ability before considering the effectiveness of MI in practice. Evaluation of MI in patients with MS requires a reliable and valid assessment tool.

To assess MI ability, researchers have developed different questionnaires such as the Movement Imagery Questionnaire (MIQ) ${ }^{12}$, the revised version of the MIQ (MIQ-R), and the Kinesthetic and Visual Imagery Questionnaire (KVIQ) ${ }^{13}$. The MIQ, developed to evaluate the MI ability of non-disabled adults and athletes, is an 18-item self-reporting questionnaire that assesses visual and kinesthetic MI ability. Later, its authors developed a shorter version, called the MIQ-R, comprising 8 items to reduce the time taken to administer the questionnaire. The results of the test-retest reliability study of the MIQ-R showed correlation coefficients of $\mathrm{r}=0.86$ for the visual subscale and $\mathrm{r}=0.90$ for the kinesthetic subscales ${ }^{14}$. Patients with neuropathology and disability displayed difficulties while using the MIQ-R because of the complexity of some movements.

Malouin et al. ${ }^{13}$ developed the KVIQ for use with both non-disabled and disabled individuals. The KVIQ assesses both visual and kinesthetic components of MI and it is not a self-administered test. All movements are assessed with the participants in a sitting position while the clarity of their imagination is rated (there is no right or wrong answer). The questionnaire has 20 items (10 items in each subscale: visual and kinesthetic) and uses a 5-point Likert scale (5=clear and intense image; $1=$ no image, no sensation) to assess the vividness of each dimension of MI (clarity of image/intensity of sensation). The results of the test-retest reliability study of the KVIQ showed intra-class correlation of 0.72-0.81 for non-disabled individuals and 0.81-0.90 for patients with stroke ${ }^{13}$. The KVIQ is suitable for people who, for whatever reason, need guidance in rating imagery and/or are unable to stand or perform physically complex movements.
To date, the test-retest reliability and internal consistency of the KVIQ have been reported in patients with stroke ${ }^{13}$ and Parkinson's disease (PD) ${ }^{13,15}$. These studies have shown that the KVIQ is a reliable and valid tool for assessing MI vividness in patients with stroke and PD. However, considering the different underlying pathologies in patients with MS and the impairment of different aspects of MI ability in patients with MS compared to those with PD and stroke (more prolonged MI in patients with PD, more prolonged and less accurate $\mathrm{MI}$ in patients with MS and less MI vividness in patients with stroke), the results of these studies cannot be directly applied to patients with MS.

In summary, this study aimed to examine: (i) the test-retest reliability and (ii) the validity of the KVIQ in patients with MS. The KVIQ was used because its reliability has been established in other neurological disorders. The MIQ-R was selected as the gold-standard test because it is short and has a high test-retest reliability ${ }^{14}$.

\section{Method}

\section{Participants}

Fifteen patients with early-stage MS (low score in the Expanded Disability Status Scale [EDSS] $)^{16}$ and aged between 20 and 40 years were included in the present study. Only those patients who satisfied the McDonald criteria ${ }^{17}$ were included in the study. All patients had neurologist-confirmed MS and were right-hand dominant (according to the Edinburgh Handedness Inventory Questionnaire) ${ }^{18}$. Patients were assessed using the EDSS. Patients with a history of other neurological diseases, head trauma, substance abuse or dependency, chronic psychiatric diseases or MS attacks in the past month were excluded. Patients were tested while on their regular medication schedule. The study was approved by the Ethics Committee of Kerman University of Medical Science, Kerman, Iran (KNRC/91/5). Written informed consent was obtained from all participants.

\section{Evaluation}

To evaluate the test-retest reliability of the KVIQ20, the same participants were assessed twice, 5-14 days apart. To examine the concurrent validity of the KVIQ-20 and MIQ-R, participants were asked to complete the MIQ-R in their second session. 


\section{Administration of the KVIQ and MIQ-R}

To promote the first-person perspective, the examiner administered the questionnaire sitting beside the subjects instead of facing them. The KVIQ was administered using the instructions outlined by Malouin et al. ${ }^{13}$. The items were presented in the same order as in the questionnaire and according to the subject's hand or foot dominance. First, the participants were asked to assume the start position. Second, they were asked to perform a described movement only once. Third, the participants returned to the starting position and imagined the movement that was just executed. Fourth, the participants were asked to rate the clarity of the visual image or the intensity of the sensations associated with the imagined movement on a 5-point ordinal scale.

On the second visit, participants were assessed by both the KVIQ and the self-administrated MIQ-R. For the MIQ-R, the participants were invited to read the instructions of the questionnaire and ask questions to the examiner if needed. The MIQ-R includes 4 movements of the trunk and the upper and lower limbs, which totaled 8 items (4 visual and 4 kinesthetic). Each item is scored according to the participant's perceived vividness ranging from 1 (very hard to see/feel) to 7 (very easy to see/feel). During the MIQ-R administration, the participants performed each movement physically once, then imagined the movement, and finally rated the vividness of each experience on the score sheet.

\section{Statistical analyses}

The consistencies of the responses of the 15 patients were investigated using test-retest analysis. After computing the total score of the KVIQ and its subscores (visual and kinesthetic) for each participant, intra-class correlation coefficients (ICCs) were measured to examine the test-retest reliability. Next, to examine the concurrent validity of the KVIQ with the MIQ-R, Spearman's correlation analysis was conducted. Furthermore, the internal consistency of the responses to the KVIQ was assessed using Cronbach's alpha. Finally, the hidden factors and components in the KVIQ were explored using factorial analysis. All these analyses were performed using the SPSS software (version 16).

\section{Results}

In all, 15 patients with a mean age of $31.73 \pm 5.52$ years were recruited. Most participants were women (12/15). The mean duration of disease was 60.8 months (minimum=12 months and maximum=144 months). Participants had a mean education of 13 years (60\% without high school diploma and 40\% with high school diploma). The mean disability score was 1.73 (minimum $=1$ and maximum $=5.5$; Table 1 ). The time interval between the test and retest sessions was 5-14 days.

The ICC was also high, ranging from 0.74 to 0.92 (Table 2).

The results of test and retest showed a high correlation between the total score on the KVIQ $(\mathrm{r}=0.89, \mathrm{P}=0.001)$ and the kinesthetic score $(\mathrm{r}=0.93$, $\mathrm{P}<0.001)$. The correlation coefficient of the visual aspect was lower than that of the kinesthetic aspect; however, it was statistically significant ( $\mathrm{r}=0.85$, $\mathrm{P}=0.001$ ).

The results of the Cronbach alpha analysis showed high internal consistency between responses to different questions (alpha $=0.84$ ). The corrected total item correlation varied from 0.44 to 0.76 .

In addition, a comparison of the score obtained from the MIQ and KVIQ showed a high correlation $(\mathrm{r}=0.79, \mathrm{P}<0.001)$. The correlation between the 2 questionnaires was also explored based on the visual and kinesthetic aspects $(\mathrm{r}=0.78, \mathrm{P}=0.001$ and $\mathrm{r}=0.75$, $\mathrm{P}=0.001$, respectively; Table 3 ).

Table 1. Patients' characteristics.

\begin{tabular}{lc}
\hline \multicolumn{1}{c}{ Variable } & Mean (SD) \\
Female/male & $12 / 3$ \\
Age (years) & $31.73(5.52)$ \\
Education (years) & $13.20(3.34)$ \\
Duration of disease (months) & $60.80(37.5)$ \\
EDSS & $1.7(1.25)$ \\
\hline
\end{tabular}

EDSS $=$ Kurtzke Expanded Disability Status Scale.

Table 2. Intra-class correlation coefficients between variables in test-retest.

\begin{tabular}{lcc}
\hline \multicolumn{1}{c}{ Variable } & ICC & P-value \\
Total KVIQ & 0.89 & $<0.001$ \\
Visual & 0.85 & $<0.001$ \\
Visual(A) & 0.74 & 0.008 \\
Visual(UL) & 0.77 & 0.002 \\
Visual(LL) & 0.89 & $<0.001$ \\
Kinesthetic & 0.93 & $<0.001$ \\
Kinesthetic(A) & 0.87 & $<0.001$ \\
Kinesthetic(UL) & 0.86 & $<0.001$ \\
Kinesthetic(LL) & 0.92 & $<0.001$ \\
\hline
\end{tabular}

$\mathrm{KVIQ}=$ Kinesthetic and Visual Imagery Questionnaire; $\mathrm{A}=\mathrm{axial}$; $\mathrm{UL}=$ upper limb; LL=lower limb. 
Table 3. Spearman correlation coefficients between the scores obtained from the KVIQ and the MIQ.

\begin{tabular}{lcc}
\hline \multicolumn{1}{c}{ Variable } & $\begin{array}{c}\text { Correlation } \\
\text { coefficient }\end{array}$ & P-value \\
Total KVIQ and total MIQ & 0.79 & $<0.001$ \\
Visual KIVQ and MIQ & 0.78 & 0.001 \\
Kinesthetic KIVQ and MIQ & 0.75 & 0.001 \\
\hline
\end{tabular}

KVIQ=Kinesthetic and Visual Imagery Questionnaire; MIQ$\mathrm{R}=$ Motor Imagery Questionnaire-Revised Version.

Finally, factorial analysis showed that there were 2 components that explained $90 \%$ of the variation; the eigenvalues were 57.6 and 32.4 for the first (visual) and second (kinesthetic) components, respectively. The first component comprised 3 variables (axial, upper limb, and lower limb) of the visual aspect. The second component also comprised 3 variables (axial, upper limb, and lower limb) of the kinesthetic aspect.

\section{Discussion}

The results of this study show that the KVIQ is a reliable tool for measuring MI ability in MS patients. In general, the reliability of the total KVIQ score was ICC $=0.89$, indicating good stability of the measures for MS patients. Moreover, the concurrent validity of the KVIQ and MIQ-R showed good-to-excellent validity $(\mathrm{r}=0.79)$.

To the best of our knowledge, the present study is the first to evaluate the reliability and validity of the KVIQ in MS patients. Our findings are consistent with those of previous studies and show similar results in patients with stroke and $\mathrm{PD}^{13,15}$.

With respect to the ICC values, the reliability was better when patients imagined movements in limbs (upper and lower) rather than axial movements, which was consistent with the results of studies in patients with stroke and $\mathrm{PD}^{13,15}$. Difficulty in axial imagery may have resulted from the small number of movements on the imagery scale of axial movements $(\mathrm{N}=3)$ compared to limb movements $(\mathrm{N}=7)$. Moreover, ICC values showed better reliability in lower limb movements than in upper limb movements during movement imagery in MS patients. Interestingly, one study showed that MS patients were confronted with higher upper limb dysfunction than lower limb dysfunction during the course of the disease ${ }^{19}$. The ICC values for the visual imagery subscale were lower than the ICC values for the kinesthetic imagery subscale. This finding is consistent with that of other studies ${ }^{13}$ and could have resulted from an ordering effect of the KVIQ subscales (visual and kinesthetic). All the visual imagery items were assessed first and kinesthetic imagery items were assessed later.

Our findings showed significant correlation between the scores for the KVIQ and MIQ-R in MS patients, which are in the good-to-excellent range, demonstrating the convergent validity of the questionnaires. The correlation coefficient in the present study was $r=0.79$, which was higher than 0.75 and was regarded as good-to-excellent ${ }^{20}$. Because of the good stability and validity of the KVIQ and its comparable results with the MIQ-R, the KVIQ can be used preferably in MS patients. In addition, in line with its bi-factorial structure, the KVIQ discriminates better between visual and kinesthetic imagery than the MIQ-R. Finally, the KVIQ is considered preferable because it is easier for disabled individuals to perform.

The high Cronbach alpha value (alpha $=0.84$ ) showed the internal consistency of the KVIQ, indicating good item homogeneity in measuring the same construct. Cronbach's alpha was high in present study and equivalent to that reported for the KVIQ in post-stroke patients ${ }^{13}$.

In the present study, factorial analysis revealed the bi-factorial structure of the KVIQ, indicating the questionnaire assesses 2 distinct components of MI (visual and kinesthetic). The 2 components explained more than $90 \%$ variation in our study, which is higher than that reported in post-stroke patients $(67.7 \%)^{13}$.

Our findings showed a high correlation $(\mathrm{r}=0.78)$ between the 2 components of the KVIQ (visual and kinesthetic), which was higher than that reported in previous studies $(\mathrm{r}=0.46)^{13}$. This finding indicates common characteristics between the 2 components.

Nevertheless, the present study has some limitations. The sample size of the study was relatively small $(\mathrm{N}=15)$, and the study was performed in a subgroup of early-stage (low disability) relapsing-remitting MS patients.

In general, our study revealed that the KVIQ can be used with good reliability and validity to assess MI ability in MS patients. With regard to the safety of movements, the KVIQ (compared to the MIQ-R) can be used to measure MI ability in disabled individuals. We recommend the KVIQ as a useful tool to assess MI ability in MS patients before adding MI to their rehabilitation program. 


\section{Acknowledgments}

This work was funded by the Kerman Neuroscience Research Center (KNRC/90/13), Kerman, Iran. We would like to thank the MS patients and the healthy controls who participated in this study and the staff members of the MS society of Kerman.

\section{References}

1. Neck CP, Manz CC. Thought self-leadership: The influence of self-talk and mental imagery on performance. J Organ Behav. 1992;13(7):681-99. http://dx.doi. org/10.1002/job.4030130705

2. Crammond DJ. Motor imagery: never in your wildest dream. Trends Neurosci. 1997;20(2):54-8. http://dx.doi. org/10.1016/S0166-2236(96)30019-2

3. Guillot A, Collet C. Contribution from neurophysiological and psychological methods to the study of motor imagery. Brain Res Rev. 2005;50(2):387-97. PMid:16271398. http://dx.doi.org/10.1016/j.brainresrev.2005.09.004

4. Ietswaart M, Johnston M, Dijkerman HC, Joice S, Scott CL, MacWalter RS, et al. Mental practice with motor imagery in stroke recovery: randomized controlled trial of efficacy. Brain 2011;134(5):1373-86. PMid:21515905 PMCid:PMC3097892. http://dx.doi.org/10.1093/brain/ awr077

5. Malouin F, Richards CL, Doyon J, Desrosiers J, Belleville S. Training mobility tasks after stroke with combined mental and physical practice: a feasibility study. Neurorehabil Neural Repair. 2004;18(2):66-75. PMid:15228801. http:// dx.doi.org/10.1177/0888439004266304

6. Tamir R, Dickstein R, Huberman M. Integration of motor imagery and physical practice in group treatment applied to subjects with Parkinson's disease. Neurorehabil Neural Repair. 2007;21(1):68-75. PMid:17172556. http://dx.doi. org/10.1177/1545968306292608

7. Heremans E, D’Hooghe M, Bondt S, Helsen W, Feys P. The relation between cognitive and motor dysfunction and motor imagery ability in patients with multiple sclerosis. Mult Scler. 2012;18(9):1303-9 PMid:22389414. http:// dx.doi.org/10.1177/1352458512437812

8. Sharma N, Pomeroy VM, Baron JC. Motor Imagery. Stroke. 2006;37(7):1941-52. PMid:16741183. http:// dx.doi.org/10.1161/01.STR.0000226902.43357.fc

9. Mulder T. Motor imagery and action observation: cognitive tools for rehabilitation. J Neural Transm. 2007;114(10):1265-78. PMid:17579805 PMCid:PMC2797860. http://dx.doi.org/10.1007/ s00702-007-0763-Z

10. Dineen R, Vilisaar J, Hlinka J, Bradshaw C, Morgan P, Constantinescu $\mathrm{C}$, et al. Disconnection as a mechanism for cognitive dysfunction in multiple sclerosis. Brain. 2009;132(1):239-49. PMid:18953055. http://dx.doi. org/10.1093/brain/awn275
11. Rocca MA, Colombo B, Falini A, Ghezzi A, Martinelli $\mathrm{V}$, Scotti G, et al. Cortical adaptation in patients with MS: a cross-sectional functional MRI study of disease phenotypes. Lancet Neurol. 2005;4(10):618-26. http:// dx.doi.org/10.1016/S1474-4422(05)70171-X

12. Hall C, Pongrac J, Buckholz E. The measurement of imagery ability. Hum Mov Sci. 1985;4(2):107-18. http:// dx.doi.org/10.1016/0167-9457(85)90006-5

13. Malouin F, Richards CL, Jackson PL, Lafleur MF, Durand A, Doyon J. The Kinesthetic and Visual Imagery Questionnaire (KVIQ) for assessing motor imagery in persons with physical disabilities: a reliability and construct validity study. J Neurol Phys Ther. 2007;31(1):209. PMid:17419886. http://dx.doi.org/10.1097/01. NPT.0000260567.24122.64

14. Lorant J, Nicolas A. Validation de la traduction française du Movement Imagery Questionnaire-Revised (MIQ-R). Sci Motricite. 2004(3):57-68.

15. Randhawa B, Harris S, Boyd LA. The Kinesthetic and Visual Imagery Questionnaire is a reliable tool for individuals with Parkinson disease. J Neurol Phys Ther. 2010;34(3):161-7. PMid:20799430. http://dx.doi. org/10.1097/NPT.0b013e3181e1aa71

16. Kurtzke JF. Rating neurologic impairment in multiple sclerosis. Neurology. 1983;33(11):144444. PMid:6685237. http://dx.doi.org/10.1212/ WNL.33.11.1444

17. McDonald WI, Compston A, Edan G, Goodkin D, Hartung HP, Lublin FD, et al. Recommended diagnostic criteria for multiple sclerosis: guidelines from the International Panel on the diagnosis of multiple sclerosis. Ann Neurol. 2001;50(1):121-27. PMid:11456302. http://dx.doi. org/10.1002/ana.1032

18. Oldfield RC. The assessment and analysis of handedness: the Edinburgh inventory. Neuropsychologia 1971;9(1):97113. http://dx.doi.org/10.1016/0028-3932(71)90067-4

19. Benedict RHB, Holtzer R, Motl RW, Foley FW, Kaur S, Hojnacki D, et al. Upper and lower extremity motor function and cognitive impairment in multiple sclerosis. Int Neuropsychol Soc. 2011;17(4):643-53. PMid:21486517. http://dx.doi.org/10.1017/S1355617711000403

20. Portney LG, Watkins MP. Foundations of clinical research: applications to practice. 2nd ed. Upper Saddle River: Prentice-Hall Inc.; 2000.

\section{Correspondence}

Nasser Zangiabadi

Kerman University of Medical Sciences Neuroscience

Research Center Kerman, Iran

e-mail: nzagiabadi1@gmail.com 